\title{
Video observation of encounters between the automated shuttles and other traffic participants along an approach to right-hand priority T-intersection
}

Petr Pokorny ${ }^{1 *}\left(\mathbb{0}\right.$, Belma Skender ${ }^{1}$, Torkel Bjørnskau ${ }^{1}$ and Marjan P. Hagenzieker ${ }^{1,2}$

\begin{abstract}
Introduction: Increasing numbers of deployment projects of automated shuttles have been taking place worldwide. Safety is one of the main concerns for their successful implementation. Therefore, it is vital to gain the knowledge about interactions between these shuttles and other traffic participants.

Method: Given the lack of behavioural observational studies under regular traffic conditions, the presented study applies external video recordings to explore encounters between the shuttles approaching a T-intersection and other traffic participants. The encounters of interest included a vulnerable road user in the bicycle lane, a pedestrian on the zebra crossing and a road user overtaking the shuttle. The shuttles were identified from the video by RUBA software. We analysed the encounters using T-Analyst software together with the manual observation of traffic participants' behaviour.

Results: From $220 \mathrm{~h}$ of video, 318 unique manoeuvres of the shuttle were observed and 83 encounters with other traffic participants were identified and explored. Several types of risks and behavioural patterns were identified, such as road users misusing the defensive style of the shuttles or cyclists in the bicycle lane not being sure about the shuttle's intention. Frequent hard stops of the shuttles might be dangerous for the passengers inside and can increase the risk of rear end accidents.

Conclusions: The findings provide a valuable insight into the interactions between automated shuttles and other traffic participants under regular traffic conditions on one location in Oslo, Norway. The study showed that introducing automated shuttles into regular traffic can lead to the emergence of new types of interactions between the shuttles and other traffic participants.
\end{abstract}

Keywords: Automated shuttle, Video observation, Encounters, Risk

\section{Introduction}

Automated shuttles (further referred as "shuttle") are low speed electrical minibuses that are able to drive without a driver. In most projects, they operate at SAE automation

\footnotetext{
${ }^{*}$ Correspondence: petr.pokorny@toi.no

${ }^{1}$ TØI - Institute of Transport Economics, Gaustadalléen 21, 0349 Oslo, Norway

Full list of author information is available at the end of the article
}

level 3 (i.e. a human operator is on board, ready to take over operation if a situation requires it) [1]. Numbers of deployment projects of these shuttles have been increasing worldwide in forms of demonstrations, pilots and showcases [2] [3]. They are typically implemented in environments such as parking lots, campuses, downtown districts, or retirement communities [4].

In Norway, public transport operators and municipalities are eager to add these shuttles into their 
regular public transport services. Since 2018, thirteen pilot projects have been carried out in ten Norwegian municipalities. They have been deployed in low-speed environments such as a shared space, a sea promenade, a pedestrian zone and a residential area, typically in places without any previous public transport. Most of pilots have taken place under regular traffic conditions on public roads. The pilots have had a variety of aims, such as testing V2X communication with traffic signals, studying the effects on travel behaviour of residents, evaluating the interactions with vulnerable road users, testing their performance in winter conditions or their suitability for an on-demand service [5].

Safety is obviously one of the biggest concerns when adding these shuttles into a complex transport system. Unexpected and new types of safety problems might emerge when these shuttles operate in regular traffic and interact with other traffic participants. Norwegian pilot projects offer a unique opportunity to explore these interactions. Given the lack of behavioural observational studies under regular traffic conditions [3], the presented study applied external video recordings to explore the encounters between the shuttles and other traffic participants along an approach to a right-hand priority T-intersection.

\section{Literature review-safety of automated vehicles}

Automated vehicles (AVs) are considered to have a potential to considerably reduce the number of road accidents [6]. This belief is founded on the assumption that human mistakes are the major contributor to the accidents and that these mistakes can be eliminated with new technologies disconnecting humans from the driving task [7]. AVs may be superior to human drivers because of better detection of other road users, better decisionmaking, or better execution [8]. However, AVs introduce additional risks into the traffic system as well $[9,10]$. Especially driving in urban environments presents one of the major challenges for AVs [11]. Here they must solve various interactions with cyclists and pedestrians, which puts considerable demands on estimation of peoples' intentions. Nevertheless, the abilities of current intention estimation algorithms are very limited [12] and pedestrians and cyclists are the most difficult to detect by AVs' sensors [13]. Furthermore, human responses to AVs are complex and not straightforward [14]. Factors such as formal and informal rules, lack of communication, miscommunication, negotiation strategies, expectations, and behavioural adaptation have an effect on interactions between AVs and other traffic participants [12, 15, 16]. A stereotypical automated driving and strict adherence to rules of AVs may cause misuse or rear end accidents [17]. In addition, there is a potential for accidents resulting from cyberattacks. Thus, AVs represent both enormous potential benefits and risks [8] and traffic safety is one of the main concerns in AVs implementation [18].

The safety performance of AVs is difficult to determine in a standard way under current conditions. The common measures of objective safety are based on the number of accidents per a unit of exposure. However, accidents are rare and AVs' mileage is still low albeit growing, often not sufficient for drawing statistically valid results $[19,20]$. Furthermore, current AVs mostly drive on the low-risk roads, making comparisons with manual driving biased [21].

Under such circumstances, using proxy indicators to accidents presents more suitable option to evaluate safety of AVs [13, 19]. These indicators can be disengagements, infractions of traffic rules or traffic conflicts. For example, a recent Australian study applied a simulation-based approach to identify conflicts by time-to-collision on a signalized intersection and a roundabout for various $\mathrm{AV}$ penetration rates [22].

Naturalistic observational studies present an alternative to evaluate safety of AVs by exploring behaviour of road users. Such approach was applied for example in a project in Oslo (NO) to evaluate the performance of automated shuttles when driving through signalised intersections [23].

Regarding specifically the safety of automated shuttles, the number of recorded accidents is obviously lower than for other types of AVs. However, when they occur, they attract considerable media and public attention. An article titled "Literally braking news: Two people hurt as not one but two self-driving space-age shuttles go awry" [24] describes two accidents. First, an incident in Utah (USA), where a shuttle detected an obstacle and stopped abruptly, causing an elderly passenger to slip off the seat. In the second accident, a shuttle in Vienna (AUT) collided with a pedestrian, who, according to witnesses, was not paying attention to the traffic. Another accident in USA, between a shuttle and a truck-tractor in Las Vegas, has been reported by NTSB [25]. In this non-injury accident, a utility refrigerated trailer was backing into an alley and struck the shuttle. The truck driver's action and his expectation that the shuttle would stop were identified as the main contributory accident factors. In August 2021, an accident occurred at the Tokyo Paralympic games. In this accident, the shuttle was turning right at a T-intersection and hit a visually impaired athlete, who was walking across the road on a pedestrian crossing. According to several newspaper articles, the shuttle was in manual mode in the moment of accident and the shuttle operator expected the athlete to stop [26, 27]. In Norway, two minor accidents have been reported so far. In 


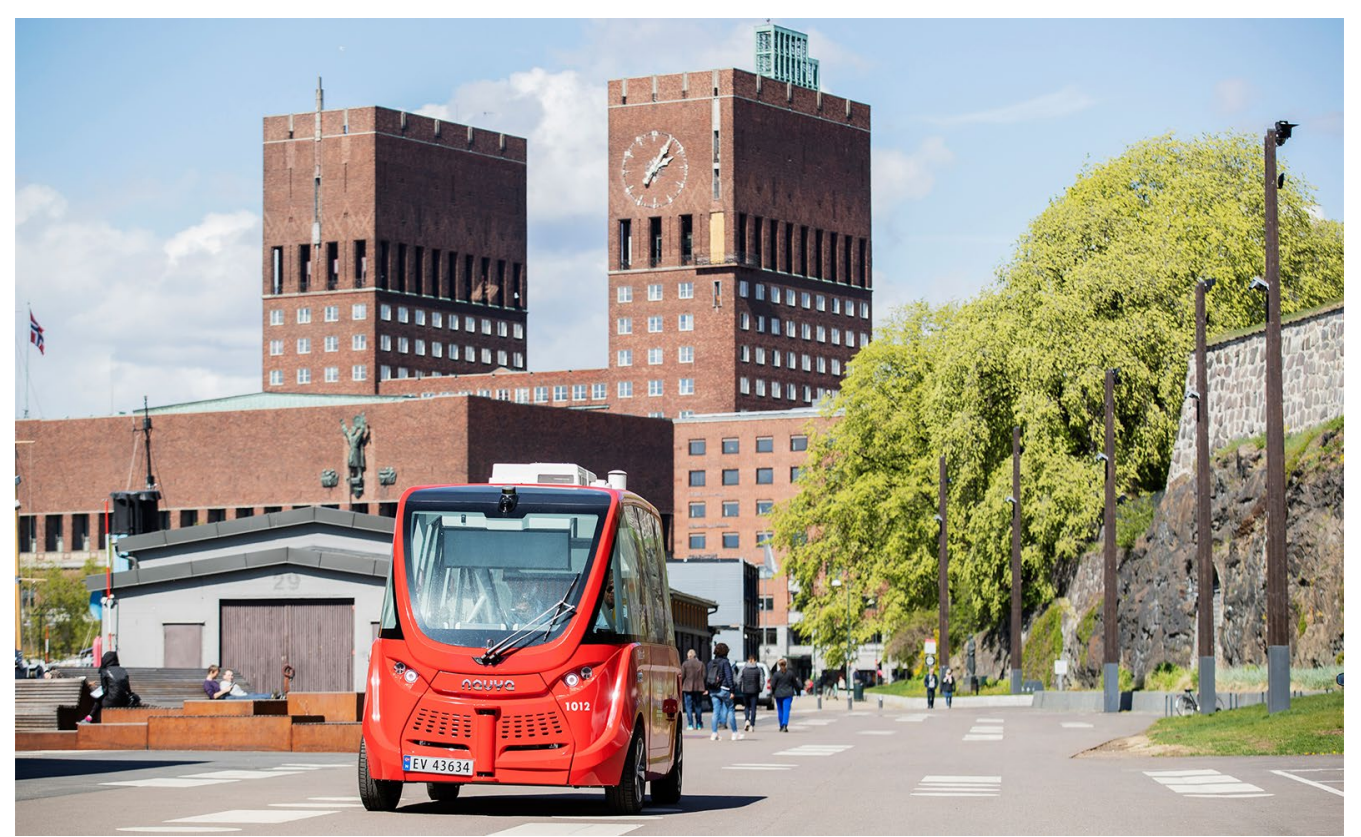

Fig. 1 One of the Navya Arma shuttles operating along Akershusstranda in Oslo ( source: https://ruter.no/om-ruter/prosjekter/selvkjorende-kjore toy/)

both accidents another vehicle was responsible for the collision [28].

Most of the studies on shuttles' safety focused on perception and acceptance of the shuttles, using surveybased methods and questionnaire-based experiments [2, 29]. For example, a survey of shuttle passengers reported that passengers assessed perception of traffic safety to be better in the shuttle than in a conventional shuttle [30]. Only a few studies applied other methods, such as the Norwegian project "Autobus" [31], studying the interactions between shuttles and other traffic participants by use of field interviews and video recordings; a case study combined with interviews to evaluate a shuttle driving on a bicycle lane [32]; workshops involving a ride in a shutthe to study the acceptability issues [33]; video analysis to study how drivers manage the traffic situations using gestures [34]; and a field study using an experimental setting and video analysis to identify policy issues and the social considerations associated with AVs [35].

\section{Method}

This study aims at exploring the encounters between the shuttles and other traffic participants along the approach to the right-hand priority $\mathrm{T}$-intersection. There is a onedirectional bicycle lane parallel the traffic lane and a raised zebra crossing on the approach. The encounter is understood as an elementary event in traffic, when participants meet at a given location at the same time. They interact in a complex way, which could be symbolically worded as "a couple coordinating their movements across the dance floor" [35]. Being affected by many factors, such dancing might go smoothly or develop into a conflict or even an accident.

In this study, the following types of encounters were explored:

- Encounters between the shuttles and vulnerable road users (VRUs) in the parallel bicycle lane. Here, the shuttle is starting to turn right and should give way to VRUs that ride parallel. Such relatively complex situation might be challenging for shuttles, while VRUs in the bicycle lane must trust that the shuttle will give way to them. Right turning encounters between larger vehicles and cyclists are considered as a particularly risky [36].

- Encounters between the shuttles and pedestrians on the raised zebra crossing. Here, the detection of pedestrians and correct estimation of their intentions might be challenging for the shuttle. In addition, pedestrians might behave differently when crossing in front of an automated vehicle [12].

- Encounters between the shuttles and other road users driving behind the shuttle. Because of strict adherence to rules and defensive driving style of the shuttles, there might be an increased risk of the shuttle being hit from behind or holding up traffic [17]. 


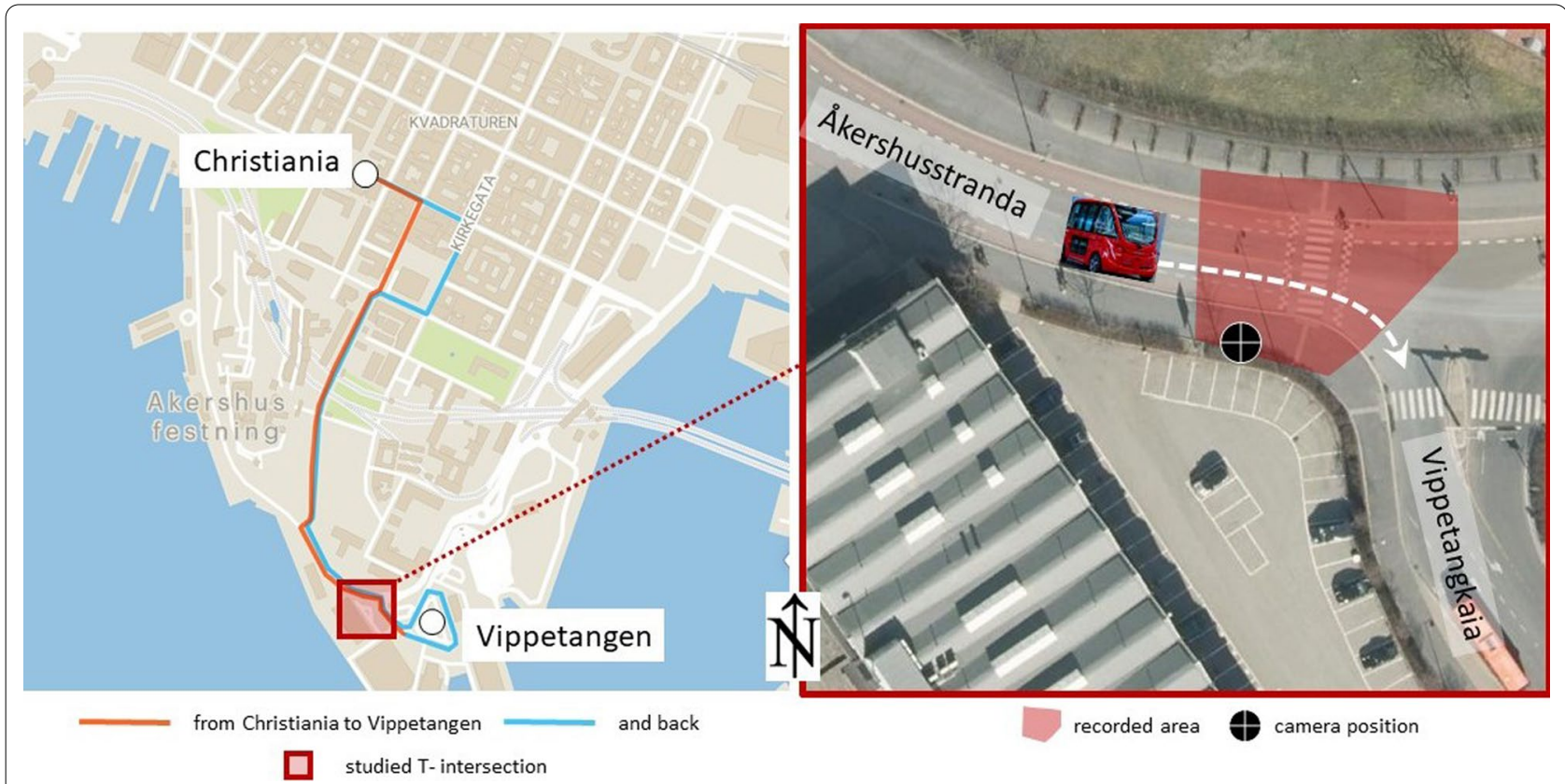

Fig. 2 The shuttle route and the location of the observed site (left); the detail of the site and the camera position (right)

The study used video recordings collected in a natural setting. Naturalistic recording is considered as one of the most effective methods for studying traffic behaviour [12]. The traffic participants and the shuttle drivers were not notified to being monitored, however some of them might have noticed the camera. We assumed that the camera had no effect on their behaviour. Video recordings were conducted with low resolution securing the anonymity of recorded traffic participants.

\subsection{The observed vehicle and site}

The type of observed shuttle was Navya Arma (see Fig. 1). In Oslo, the local public transport provider Ruter has been testing these shuttles since 2019 as an integrated part of Oslo region's public transport network. ${ }^{1}$

Parameters and technological equipment of these shuttles are:

- Capacity: 8 passengers

- SAE level: 3

- Max speed: $18 \mathrm{~km} / \mathrm{h}$

- Dimensions: $4.75 \mathrm{~m}$ long, $2.11 \mathrm{~m}$ wide and $2.65 \mathrm{~m}$ tall

- LIDAR (4 front and 4 back sensors) for detecting objects, obstacles, and landmarks within an established radius around the shuttle

\footnotetext{
${ }^{1}$ The total number of vehicle $\mathrm{km}$ driven by these shuttles has exceeded $33,000 \mathrm{~km}$, and the total number of passengers reached 29,000 (status in December 2020; https://ruter.no).
}

- Global Navigation Satellite System for providing positioning, navigation, and timing

- Odometer for measuring the distance travelled

- An inertial measurement unit which measures acceleration, orientation, angular rates, and other gravitational forces

To follow their pre-defined trajectory and solve a variety of traffic situations, the shuttles are equipped with pretrained models, which are not self-learning. Therefore, they could fail if facing a situation, they have not been trained for [28]. During the course of deployment projects, software updates are periodically implemented to adjust the shuttle's performance.

The location for observation was selected on the basis of a previous work conducted within TØI project Autobus [31]. Here, on its way from the Christiania square towards Vippetangen, the shuttle was approaching the right-hand priority $\mathrm{T}$-intersection and was turning right from Akershusstranda into Vippetangkaia (see Fig. 2).

Speed limit changes from $30 \mathrm{~km} / \mathrm{h}$ to $40 \mathrm{~km} / \mathrm{h}$ on Akershusstranda. This change is indicated by a traffic sign placed about $20 \mathrm{~m}$ before the T-intersection (i.e. the recorded section has $30 \mathrm{~km} / \mathrm{h}$ speed limit). There is raised zebra crossing on the approach and one-directional bicycle lanes (red surface; $2.1 \mathrm{~m}$ wide) along both sides of Akershusstranda. The right-turning vehicles must yield to cyclists/e-scooters who are riding straight in the bicycle lane (along the right of a turning vehicle). 
Table 1 Traffic volumes from 09.30 to 20.30 for a typical working and weekend day

\begin{tabular}{llllll}
\hline & \multicolumn{2}{l}{$\begin{array}{l}\text { Bicycle lane and traffic } \\
\text { lane (one direction) }\end{array}$} & & \multicolumn{2}{l}{$\begin{array}{l}\text { Zebra crossing (both } \\
\text { directions) }\end{array}$} \\
\cline { 2 - 3 } \cline { 5 - 6 } \cline { 5 - 6 } & $\begin{array}{l}\text { Cyclists and } \\
\text { e-scooterists }\end{array}$ & $\begin{array}{l}\text { Motorised } \\
\text { vehicles }\end{array}$ & & Pedestrians & $\begin{array}{l}\text { Cyclists and } \\
\text { e-scooterists }\end{array}$ \\
\hline Working day & 974 & 1151 & & 118 & 39 \\
Weekend day & 738 & 700 & & 171 & 109 \\
\hline
\end{tabular}

The shuttles were in operation from June to September 2020. They operated every week from Thursday to Sunday, from 09.30 till 20.30. They were scheduled in 30 min' intervals, however due to operational and external factors (such as a road maintenance), their daily numbers varied. During the observational period the number of shuttles varied daily from 8 to 24 (the mean value of 16; standard deviation 5.5 ). They mostly operated between $10.00-15.00$, without any difference between working and weekend days. According to the shuttle operator company, the shuttles were driving in automated mode during the studied period.

The traffic volumes were calculated from the video using RUBA software [37]. Cyclists (including e-scooters) and motorised vehicles were counted in the similar traffic direction as the shuttle. Traffic participants using the zebra crossing were counted in both directions. The counts were conducted for both working and weekend days (10/09/2020-Thursday; 19/09/2020-Sunday) from 09.30 to 20.30 (see Table 1). In both days, the weather was nice, warm and sunny.

During the working days, there were obvious peak times in the afternoon (14.30-15.30 for motorised vehicles and 15.30-17.30 for cyclists and e-scooterists), while during the weekends, the traffic was more evenly distributed throughout the day. The traffic can be characterised as a mixture of recreational, sport and commuting.

\subsection{Video data-collection}

One portable Miovision Scout camera unit (attached to a lighting pole) recorded the site for twenty days (6-9/8; 13-16/8; 3-4/9; 10-11/9; 17-20/9; 24-27/9). The recording was conducted from $6.0 \mathrm{~m}$ height, with the resolution $720 \times 480$ pixels and speed $30 \mathrm{fps}$ (see Fig. 2 for the camera position and the recorded area). The camera angle was slightly adjusted after five days of recording $(3 / 9 / 2020)$ in order to capture a longer section of the shuttle's approaching manoeuvre (see the difference in Fig. 3).

To detect the shuttles in the video, a presence detector created in RUBA software was placed in the video recordings in such position and with such benevolent parameters that it detected all right-turning road users. RUBA provided a picture and time of every detection and those containing the shuttle were manually filtered out in a generic photo-viewer. According to the detection times, the relevant video sequences were viewed and those containing an encounter between the shuttle and another traffic participant were cut into separate video clips (using Windows Picture app).

\subsection{Video data-analyses}

The video clips were analysed by an experienced road safety researcher. The numerical measurements (i.e. speeds, accelerations, positions, trajectories) were conducted in T-Analyst software [38]. To account for the distortion of the camera, the recordings were calibrated in T-calibration software [38] by connecting points in the camera view to the real world points, using aerial image with known scale from www.finn.no (see Fig. 4). In addition, the shuttle's speed data in several encounters were obtained from the shuttle operator, so it was possible to compare the values with speed measured in T-Analyst.

In order to get a reference speed and trajectory of an "uninfluenced" shuttle under free flow traffic conditions (i.e. not experiencing any encounter), a sample of five randomly selected shuttles (captured by the camera in angle \#2) was used. To measure a reference speed of straight driving passenger cars and straight riding cyclists under free flow traffic conditions, two random samples of 100 vehicles and 100 cyclists were selected and their speeds were measured manually from the video (when driving/riding over the zebra crossing). Motorised vehicles driving behind the shuttle and having the opportunity to overtake (i.e. no traffic in opposite direction and nobody in the proximity of the pedestrian crossing) were manually counted from the video clips.

For the measurements of the road users' positions, the coordinates $[0 ; 0]$ were set to the point, where the bicycle lane marking meets the marking of the raised pedestrian crossing (see Fig. 5). In time $t=0$ (when it was possible to start tracking the shuttle in T-Analyst), the front of the shuttle was $8.7 \mathrm{~m}$ away from that point (in camera angle \#2), respective $6.2 \mathrm{~m}$ in camera angle \#1. The total length of the observable section was $15.0 \mathrm{~m}$ (measured from the front of the shuttle in $t=0$ to the back of the shuttle in the very last moment it was possible to track its trajectory).

In addition to the numerical measurements, the following data were collected for every encounter:

- Time and date of the encounter

- The type and number of involved traffic participants (if there were several encounters between one shuttle and other traffic participants, these were distinguished as primary and secondary encounters) 

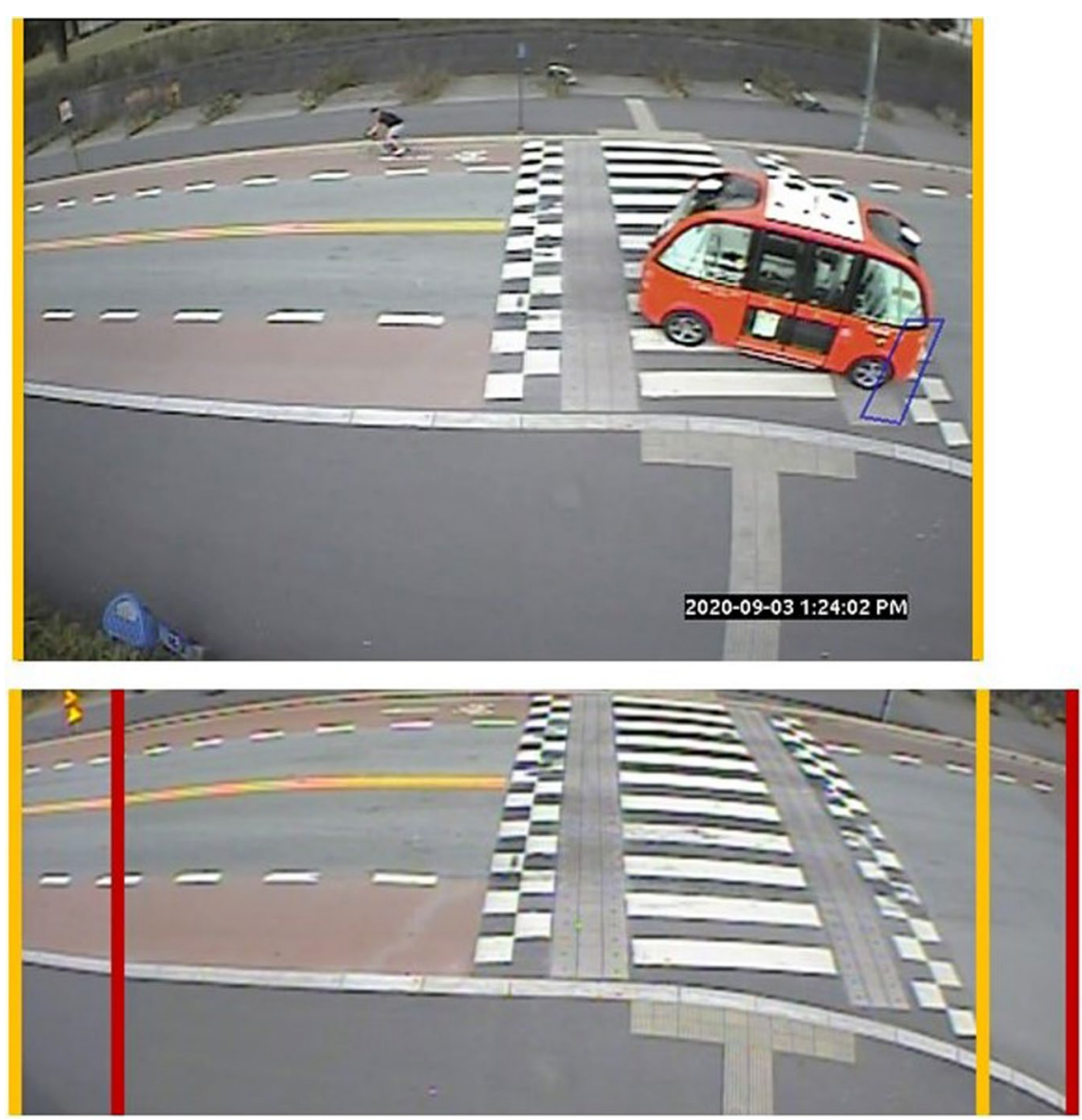

\section{Angle \#1}

Angle \#2

Fig. 3 The original angle of the video recording (red) and the adjusted angle (yellow)

- Complying with the traffic rules

- The context of the encounter (simple= predictable behaviour, mostly just one other traffic participant presented; less simple $=$ several traffic participants moving in different directions in the proximity of the shuttle and/or a traffic participant behaving in unexpected manner). Encounters, when a road user was overtaking the shuttle, were considered as simple.

- A brief description of the encounter (including driving directions and actions of involved traffic participants).

The aforementioned measurements and variables enabled to explore the encounters, identify the behavioural patterns and potential risks.

\section{Results}

In total $220 \mathrm{~h}$ of video were collected during 20 days of recording. From these, 318 unique manoeuvres, when the shuttle was approaching the intersection and turning right, were detected. Sixty-five of them included at least one encounter with another traffic participant.

\subsection{Standard manoeuvres and speeds}

Under free flow traffic conditions, the shuttle was approaching the $\mathrm{T}$-intersection with the speed of $10.0 \mathrm{~km} / \mathrm{h}$. Approximately $4.0 \mathrm{~m}$ in front of the zebra crossing the shuttle started slowing down steadily, reaching the minimum speed of $6.0 \mathrm{~km} / \mathrm{h}$ when entering the zebra crossing. During the turning manoeuvre the shuttle accelerated to ca. $8.0 \mathrm{~km} / \mathrm{h}$. Figure 6 illustrates the reference speed profile of such standard manoeuvre.

Under free flow traffic conditions, the mean speed of straight driving passenger cars was $25.7 \mathrm{~km} / \mathrm{h}$ (standard deviation $5.1 \mathrm{~km} / \mathrm{h}$ ). The mean speed of straight riding uninfluenced cyclists in the bicycle lane was $20.9 \mathrm{~km} / \mathrm{h}$ (standard deviation $4.0 \mathrm{~km} / \mathrm{h}$ ).

\subsection{Encounters between the shuttle and other traffic participants}

There were in total 83 encounters identified in 65 video clips. Most of them $(\mathrm{n}=71,85.5 \%)$ occurred within 

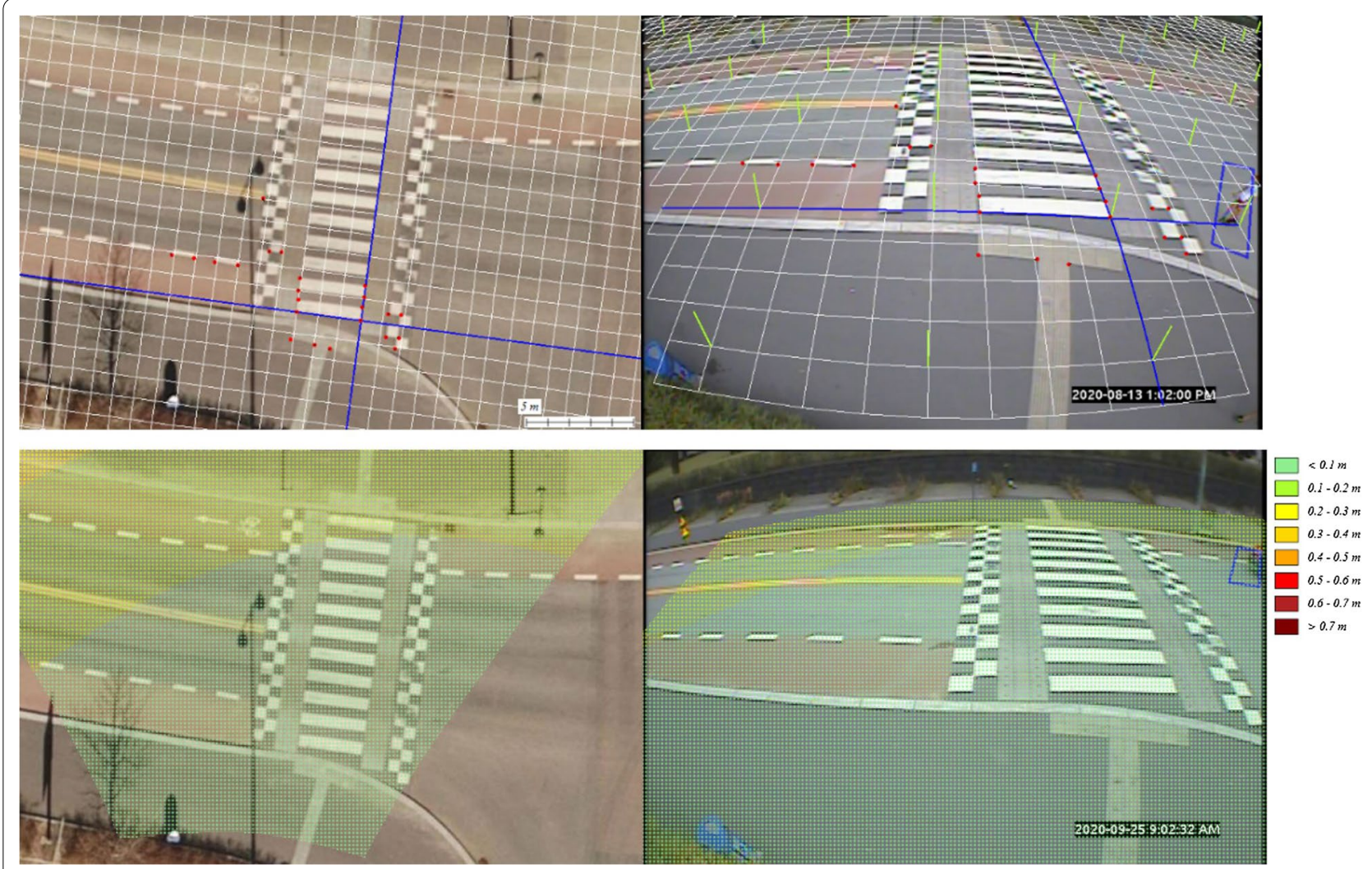

Fig. 4 Calibration grid and the camera accuracy

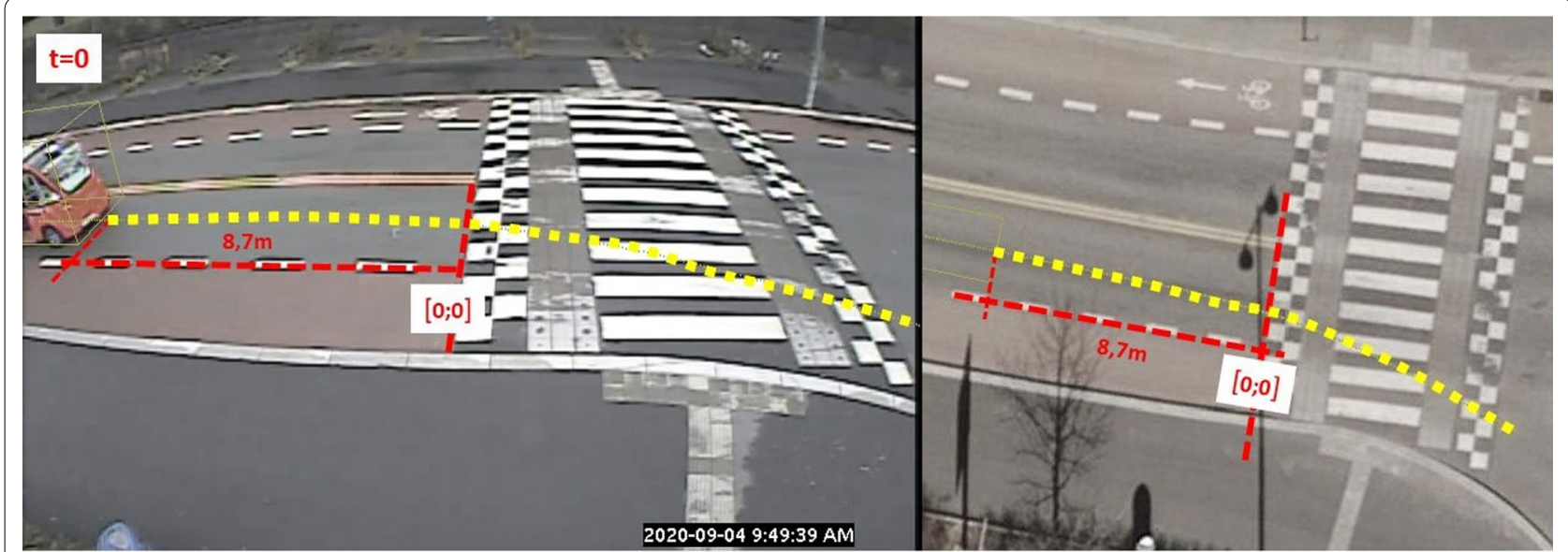

Fig. 5 The first traceable position of the shuttle in camera angle \#2 $(t=0$, distance $=8.7 \mathrm{~m})$, the axes for distance measurements (red lines) and the trajectory of the shuttle (yellow line)

a simple context. Three types of encounters were recognised:

- a vulnerable road user in the bicycle lane $(n=60)$

- a pedestrian on the zebra crossing $(n=4)$

- a road user overtaking the shuttle $(\mathrm{n}=19)$

\subsubsection{A vulnerable road user in the bicycle lane $(n=60)$}

There were 45 primary and 15 secondary encounters with a VRU in the bicycle lane. Primary encounters involved 35 cyclists, 9 e-scooterists and 1 jogger. Secondary encounters involved 11 cyclists and 4 e-scooterists. 


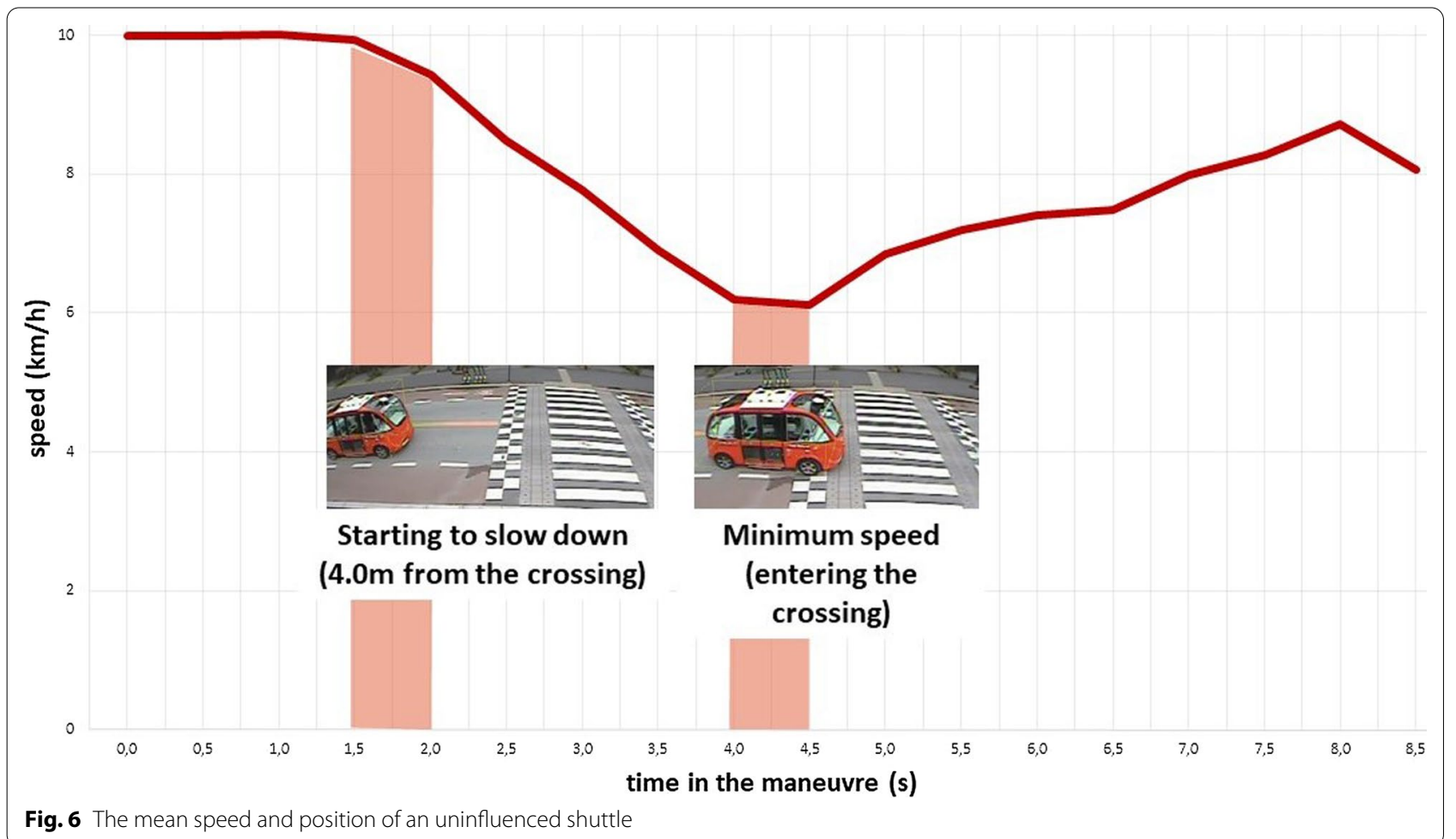

4.2.1.1 Primary encounters The reactions of the shuttle in the primary encounters can be categorised as "slowing down without a stop" $(\mathrm{n}=22)$, "hard stop" ${ }^{2}(\mathrm{n}=17)$, "steady stop" ( $\mathrm{n}=5)$ and "no reaction" $(\mathrm{n}=1)$.

In almost all "slowing down without a stop" encounters, the shuttle was starting to slow down outside the camera angle, reaching the minimum mean speed of $3.2 \mathrm{~km} / \mathrm{h}$ (standard deviation $1.4 \mathrm{~km} / \mathrm{h}$ ). The mean deceleration was $-0.51 \mathrm{~m} / \mathrm{s}^{2} \quad(\mathrm{n}=14$, standard deviation $0.24 \mathrm{~m} /$ $\mathrm{s}^{2}$ ). The mean speed of VRUs (when riding parallel to the shuttle) was $22.2 \mathrm{~km} / \mathrm{h}(\mathrm{n}=17$, standard deviation $4.8 \mathrm{~km} / \mathrm{h}$ ). In 11 encounters, the VRU was increasing the speed, while in 6 encounter the speed was constant. In one encounter the shuttle slowed down as a reaction to a group of cyclists standing on the sidewalk.

The encounters resulting in the "hard stops" were characterised by the shuttle's deceleration from the mean speed of $8.4 \mathrm{~km} / \mathrm{h}$ (standard deviation $0.9 \mathrm{~km} / \mathrm{h}$ ) to full stop during less than $1.5 \mathrm{~s}$. The mean deceleration was $-1.61 \mathrm{~m} / \mathrm{s}^{2}$ (standard deviation $0.44 \mathrm{~m} / \mathrm{s}^{2}$ ). Hard stops typically occurred close to the zebra crossing (the mean stopping position was $1.3 \mathrm{~m}$ in front of the crossing). When the shuttle started to decelerate, a VRU in the bicycle lane was typically located several meters behind

\footnotetext{
${ }^{2}$ A «hard stop» was defined as a sudden, obvious, intense break (when compared with the standard maneuver), recognizable from both the video and speed/acceleration data in T-Analyst.
}

the shuttle, outside the camera angle. The mean speed of VRUs (when riding parallel to the shuttle) was $16.5 \mathrm{~km} / \mathrm{h}$ $(\mathrm{n}=15$, standard deviation $5.1 \mathrm{~km} / \mathrm{h})$. In 12 encounters, VRUs kept a constant speed during the whole encounter, in three encounters VRUs slightly increased the speed. Two encounters resulted in a "stalemate situation", when both the shuttle and VRU stopped, probably not being sure about the other's intentions. After a few seconds, the shuttle continued first. When observing the movements inside the shuttle, it was obvious that the shuttle operator took over the driving to solve these stalemate situations.

In "steady stops", the shuttle was starting to slow down outside the camera angle. Therefore, its mean speed in the initial tracking position was already relatively low (5.9 km/h, standard deviation $2.3 \mathrm{~km} / \mathrm{h}$ ). The mean stopping position was $1.8 \mathrm{~m}$ in front of the crossing. The mean deceleration was $-0.4 \mathrm{~m} / \mathrm{s}^{2}$ (standard deviation $0.2 \mathrm{~m} / \mathrm{s}^{2}$ ). In two encounters it was possible to measure the speeds of VRUs (cyclists riding in $10.1 \mathrm{~km} / \mathrm{h}$ and $16.2 \mathrm{~km} / \mathrm{h}$ ). One encounter (with e-scooter) developed into a stalemate situation. In another encounter, the shuttle stopped, while a couple of cyclists were standing still in the bicycle lane. In the last encounter, an e-scooterist moved away from bicycle lane onto the sidewalk.

Figure 7 shows the course of the shuttle's deceleration during a hard stop and a steady stop. 


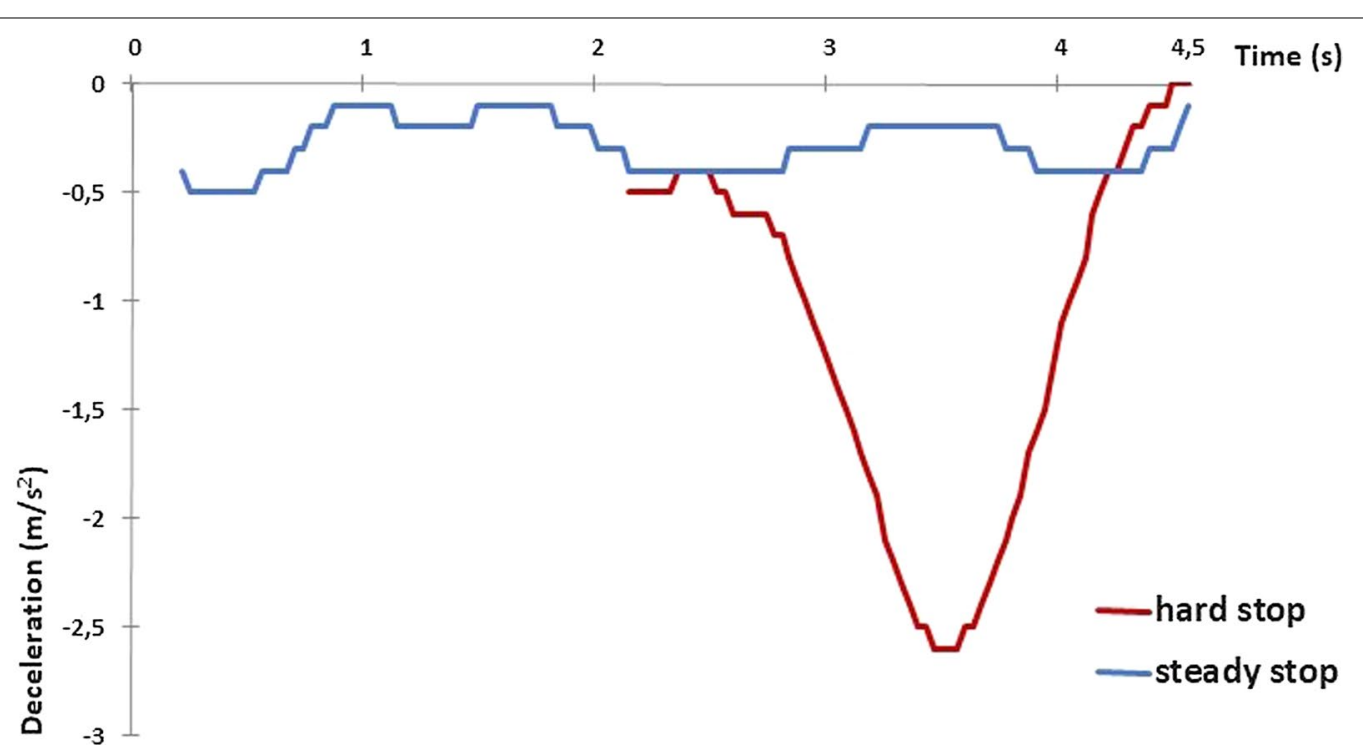

Fig. 7 The example of shuttle's deceleration during a hard stop and a steady stop

There was only one encounter without "any reaction" of the shuttle. In this encounter the shuttle was turning right, while an e-scooter (that was riding several meters behind the shuttle) slightly slowed down. The reaction of the e-scooter was very gentle (not evasive) and the encounter could be considered to be in accordance with the traffic rules.

4.2.1.2 Secondary encounters In the secondary encounters, the shuttle had already stopped $(\mathrm{n}=9)$ or was moving very slowly again after a primary encounter (mean speed of $2.7 \mathrm{~km} / \mathrm{h}, \mathrm{n}=6$ ), when a VRU approaching from behind triggered a secondary reaction. The mean speed of the secondary VRUs, measured in the position parallel to the shuttle, was $17.7 \mathrm{~km} / \mathrm{h}$ (standard deviation $6.0 \mathrm{~km} / \mathrm{h}$ ). $60 \%$ of VRUs were keeping the constant speed, while the rest was accelerating. The mean time gap between the primary and secondary VRU was $4.3 \mathrm{~s}$ (standard deviation $3.1 \mathrm{~s}$ ). In all encounters when the shuttle had already stopped by a primary encounter, it stayed stopped in the secondary encounter. In the remaining six encounters (when the shuttle was slowly moving), the shuttle stopped again in four cases, and in two cases it slowed down. In two situations, that involved multiple secondary encounters, it was obvious from the observable movements inside the shuttle that the shuttle operator overtook the control over the driving.

\subsubsection{A pedestrian on the zebra crossing $(n=4)$}

There were four encounters with pedestrians. In all of them, the shuttle was approaching the zebra crossing in its full speed and a pedestrian was approaching the
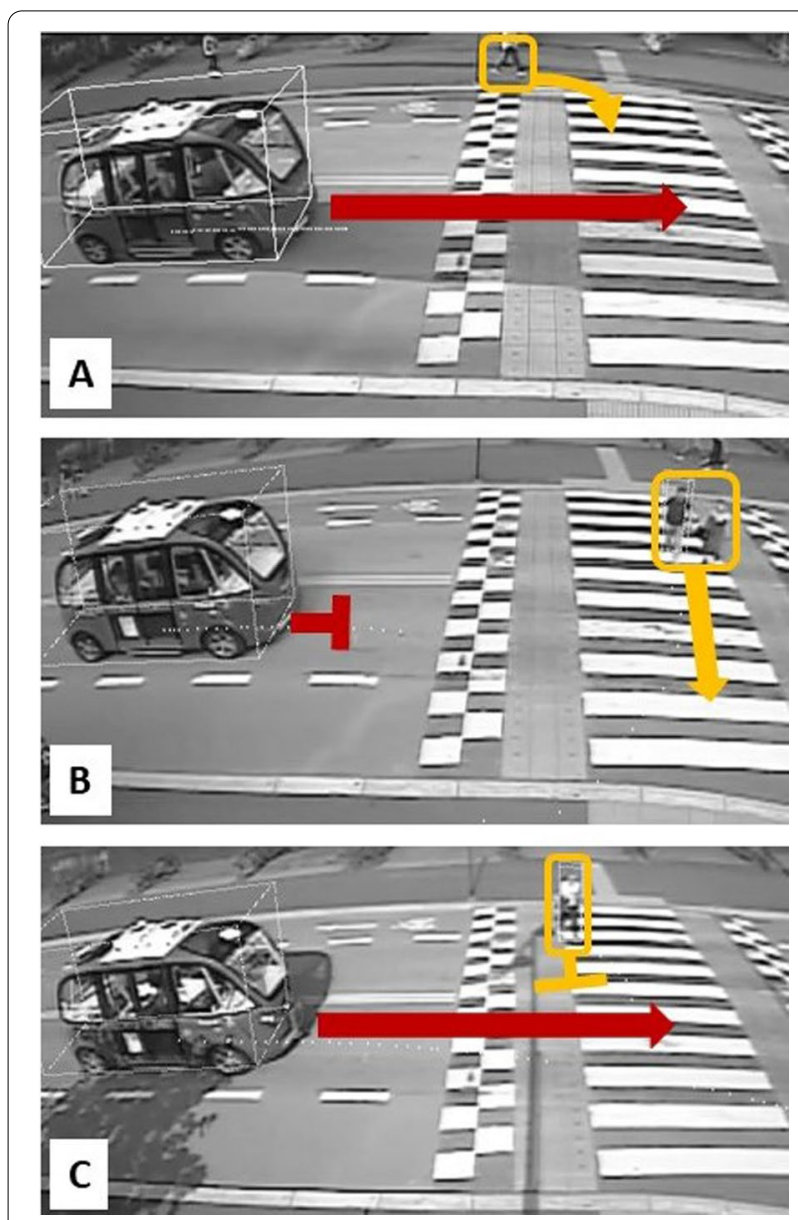

Fig. 8 The examples of encounters between the shuttle and pedestrians. a The shuttle does not react; $\mathbf{b}$ the shuttle yields, but stops too early; $\mathbf{c}$ the shuttle does not give a way 
crossing from the left (from the view of the shuttle). In one encounter, the shuttle correctly did not yield, since the pedestrian was still in a sufficient distance from the crossing (Fig. 8a). In the second encounter, the shuttle correctly yielded, however it stopped too far from the crossing (Fig. 8b). In the remaining two encounters, the shuttle should have yielded, but it did not, and the pedestrian had to either stop or slow down to let the shuttle drive (Fig. 8c).

\subsubsection{A road user overtaking the shuttle $(n=19)$}

We observed 116 situations where a motorised vehicle was driving behind the shuttle and there was simultaneously no on-coming traffic in the opposite traffic lane and no pedestrian near the crossing. Such a set-up presents an opportunity to overtake the shuttle, and we observed that. Sixteen vehicles (13.8\%) overtook the shuttle in this situation. Most were passenger cars $(n=14)$, one was a truck and one was a motorcycle. In thirteen of these encounters the shuttle was moving with its standard speed (ca $10.0 \mathrm{~km} / \mathrm{h}$ ). To perform the overtaking manoeuvre, the road users had to cross the solid centre lane marking, drive in the opposite traffic lane and overtake the shuttle over the raised pedestrian crossing. Such manoeuvre is not according to the traffic rules. The shuttle did not react to the overtaking vehicles except in one situation when it slowed down while being overtaken by three vehicles in a row. In three overtaking encounters the shuttle had already stopped as result of another encounter.

Furthermore, there were two cyclists and one e-scooterist observed to overtake the shuttle. Both cyclists were sport cyclists riding fast $(25.0 \mathrm{~km} / \mathrm{h})$. In the first encounter the shuttle did not react to the overtaking cyclist who was riding in the traffic lane the whole time. In the second encounter the shuttle stopped hard because the cyclist, who started to overtake from the bicycle lane, rode too close to the shuttle. In the situation where the e-scooterist overtook the shuttle, the shuttle had already stopped as result of another encounter.

\section{Discussion}

The emphasis of the study was to explore the encounters between the shuttles and other traffic participants and identify potential risks. Because of low speeds of the shuttles, encounters' characteristics and mutual trajectories of the shuttles and traffic participants (mostly moving parallel to each other or behind each other), it was not feasible to measure time-to-collision or postencroachment time as traffic safety indicators. Rather, we looked after observable evasive manoeuvres, compliance to traffic rules, abrupt stops and changes in speeds. We did not use the shuttles' internal operational data for the measurements, but instead we measured these variables externally in T-Analyst software, as we did for other traffic participants. However, we compared the T-Analyst measurements of speed and deceleration of several shuttles with their internal data and the results were similar. We discuss the findings separately for the three encounter types.

\subsection{Encounters with vulnerable road users in bicycle lane}

All encounters were observed along the approach to the T-intersection, before the shuttle's actual right turning manoeuvre. The shuttles performed well in encounters where VRUs behaved in a predictable/standard manner and were not too close to the shuttle's trajectory/safety zone.

However, in regular traffic the traffic participants often ride close to the shuttle and behave differently than the shuttle is programmed to be prepared for. Thus, 37\% of primary encounters between the shuttle and a VRU in the bicycle lane resulted in a hard stop by the shuttle (Additional file 1). None of the observed hard stops was performed as an obvious evasive manoeuvre (i.e. to prevent an accident) and can be considered as false alarms [13]. The hard stops are recognised as a problem of AV shuttles [23]. They are typically triggered by another road user being detected within the shuttle's safety zone, while the exaggerated intensity of these stops is of a technological nature. On the studied site, the hard stops typically occurred closer to the zebra crossing than the "gentler" reactions, and they were characterised by the mean deceleration of $-1.61 \mathrm{~m} / \mathrm{s}^{2}$. VRUs involved in hard stops were riding slower than VRUs involved in the gentler encounters or in uninfluenced situations. This might indicate that VRUs were not sure about the shuttle's intentions and therefore were reducing their speed. Hard stops can be considered as potentially risky, both for passengers inside the shuttle and for the drivers behind the shuttle. We observed movements characterized by uncontrolled loss of balance of passengers inside the shuttle as a result of hard stops. In order to warn the passengers about this issue, there is even a warning sigh inside the shuttles. As for the drivers behind the shuttle, the unexpected hard stops might increase the risk of rearend accidents, that have been identified in several studies as a common accident type related to AVs [39].

Encounters in which VRUs behaved differently than the shuttle was programmed to recognize, such as those involving a cyclist standing still in the bicycle lane and talking with a person standing on the sidewalk (Additional file 2) or e-scooters moving from bicycle lane onto the sidewalk, were wrongly interpreted by 
the shuttle and triggered unnecessary reactions by the shuttle (i.e. slowing or stopping). Similar issues were recognised at signalised intersections in Oslo [23] or during a pilot in a medical campus-type setting [35].

Four encounters were linked to a hesitation of VRUs to undertake the shuttle, which resulted in a "stalemate" situation, when both the shuttle and the VRU stopped (Additional file 3). The shuttle driver took over the driving to solve these situations and finished the turning manoeuvre, driving before the VRU, despite that the VRU should ride first. Although these encounters could be considered as not complying with the traffic rules, the shuttle driver solved the situation in a safe way. These encounters indicate that the shuttle's intentions might not be clear to some road users.

\subsection{Encounters with pedestrians on zebra crossing}

There were four encounters observed on the zebra crossing. From such small sample, it is not possible to make a reliable conclusion. However, in two encounters the shuttle did not give way to a pedestrian approaching from the left (Additional file 4). According the shuttle's operator, the shuttle was not programmed to give way in these situations. As pedestrians have right of way in this type of encounter, such programming failure increases the accident risk.

\subsection{Encounters with road users in traffic lane behind the shuttle}

When drivers of following motorised vehicles had the opportunity to overtake the shuttle, almost $14 \%$ of them decided to overtake (Additional file 5), even if such a manoeuvre is not legal on this location. This willingness to break the traffic rules may be explained as an effort to avoid a potential delay caused by the speed difference between the passenger cars and the shuttle (the mean speed of the cars under free flow condition was almost three times higher than the shuttle's speed). The reason for overtaking might also be to reduce the exposure to the defensive and stereotypical driving of the shuttle. Similar overtaking behaviour has been observed at other locations as well [31]. Such overtaking might be potentially risky for pedestrians stepping at the zebra crossing from the right side, since overtaking drivers would have limited visibility because of the shuttle. Obviously, overtaking can also be a risk factor for oncoming traffic. However, such particular situations were not observed in this study. The shuttle did not react to overtaking vehicles, probably because the overtaking cars were not too close to its pre-defined trajectory. However, on other locations along the straight sections in Oslo the shuttles frequently reacted (stopped) to overtaking cars [31].

\subsection{Identified risks}

To summarise, the following types of risk were recognised in the observed encounters:

1. Risk for passengers to falls inside the shuttle as the result of unexpected and exaggerated reactions (hard stops) of the shuttle.

2. Risk of rear-end accidents for motorized vehicles that are driving closely behind the shuttle at the moment of an unexpected or exaggerated stop of the shuttle.

3. Risk for pedestrians on a zebra crossing and for oncoming traffic because of motorized vehicles overtaking the shuttle.

4. Risk for pedestrians approaching a zebra crossing from the left because the shuttle was not programmed to give way in this scenario.

\subsection{Limitations}

A major limitation of observational studies is the observer bias, which are caused by both the observer's misperception and subjective judgment of the traffic situations [12]. In this study, several quantitative variables were measured, which should reduce this bias. Another limitation of external video observation is the impossibility to reliably recognize underlying motives, intentions or expectations of observed traffic participants. Within this study it is for example not possible to recognize, whether the cyclists did not know the traffic rules or they had limited trust in the shuttle's intentions in the observed stalemate situations. To remedy this issue, a hybrid approach combining observations with on-site interviews would be necessary [12]. However, such approach was out of scope in this study.

There was also a limitation related to the size of the recorded/observed area. This area covered a $15.0 \mathrm{~m}$ long approach section to the T-intersection and the zebra crossing on the approach. In several encounters that were initiated further from the T-intersection, it was not possible to measure the initial positions and speeds of observed traffic participants. This limitation was due to the camera angle (max. $120^{\circ}$ horizontal view) and because there was only one suitable nearby lighting pole to attach the camera to.

The small sample size presents another limitation of this study. However, the unique possibility to explore the encounters under regular traffic conditions compensates this shortcoming.

In addition to that, the shuttle's behaviour and reactions are determined by its pre-trained models and safety/priority zones. Knowledge of these models on the observed location would have made the analyses of encounters more accurate (from the shuttle's 
perspective). Unfortunately, we had only limited access to this information.

\section{Conclusion}

An introduction of automated shuttles into regular traffic leads to the emergence of new types of interactions, conflicts and possibly accidents. Therefore, the successful implementation of these shuttles will not be possible without a deeper understanding of their interactions with other traffic participants in regular traffic. This study, despite its simplicity in terms of limited number of observations and safety indicators, provides a relevant piece of knowledge into this understanding. Using video observations, we identified several potential risks in encounters between the shuttles and other traffic participants, such as risks related to abrupt stops of the shuttles, the shuttles not giving way to pedestrians on the zebra crossing or dangerous overtakings of some road users as reaction to the shuttles' defensive and slow driving style. Furthermore, the human operator had to take over control to solve a variety of situations occurring in regular traffic. When interpreting the results, the local conditions, technical parameters and software setup of the shuttles, together with the methodological limitations must be considered.

Our findings suggest, that there is still lot of work required before the shuttles can safely and smoothly operate in regular complex urban traffic without a human operator on board. On the other hand, public transport operators and municipalities are eager to add these shuttles into their regular public transport services, especially in areas without existing public transport services and for on-demand services. The pace of the technology development is enormous, and the existing technological problems will probably be solved in a very near future. Therefore, the safety research must closely follow the rapid development of automated shuttles and their deployments into traffic. As for the shuttles, the crucial technological and software challenges are the correct detection and interpretation of traffic situations, intensity of braking, and communication with other road users. The research must focus not only on the shuttles, but also on how other traffic participants perceive them, how they behave when encountering the shuttles, and why they behave in such ways [5].

\section{Supplementary Information}

The online version contains supplementary material available at https://doi. org/10.1186/s12544-021-00518-x.

Additional file 1. A hard stop of the shuttle.
Additional file 2. The shuttle reacts to cyclists standing in the bicycle lane.

Additional file 3. An example of the stalemate situation.

Additional file 4. The shuttle does not yield to the pedestrian coming from left.

Additional file 5. A truck is overtaking the shuttle.

\section{Authors' contributions}

All authors initiated the study and developed its design. PP conducted the study and prepared the draft of the manuscript. BS processed the video recordings and took part in scientific discussion over the findings, MH and TB provided comments and edited the manuscript. All authors worked together on the final version of the manuscript. All authors read and approved the final manuscript.

\section{Funding}

The manuscript has been produced within Drive2theFuture project (funded under the European Union's Horizon 2020 Research and Innovation Programme, Grant Agreement No. 815001), together with an internal research grant from TØl.

\section{Availability of data and materials}

All documents analysed during this study are referenced in this published article.

\section{Declaration}

\section{Competing interests}

The authors declare that they have no competing interests.

\section{Author details}

${ }^{1} \mathrm{~T} \varnothing \mid$ - Institute of Transport Economics, Gaustadalléen 21, 0349 Oslo, Norway. ${ }^{2}$ TU Delft - Delft University of Technology, Delft, The Netherlands.

Received: 9 December 2020 Accepted: 11 November 2021

Published online: 25 November 2021

\section{References}

1. SAE International. (2018). Taxonomy and definitions for terms related to driving automation systems for on-road motor vehicles. Standard J3016201806. Retrieved October 25, 2020, from https://www.sae.org/stand ards/content/j3016_201806/

2. Heikoop, D. D., Velasco, J. P. N., Boersma, R., Bjørnskau, T., \& Hagenzieker, M. P. (2020). Automated bus systems in Europe: A systematic review of passenger experience and road user interaction. Advances in Transport Policy and Planning, 5, 51-71. https://doi.org/10.1016/bs.atpp.2020.02.001

3. Haque, A. M., \& Brakewood, C. (2020). A synthesis and comparison of American automated shuttle pilot projects. Case Studies on Transport Policy, 8, 928-937. https://doi.org/10.1016/j.cstp.2020.05.005

4. Valdes, V., Torn, G. W., Mortensen, S., \& Diggs, D. (2018). Strategic transit automation research plan. Retrieved July 18, 2020, from https://www.trans it.dot.gov/automation-research

5. Pokorny, P., Skender, B., \& Bjørnskau, T. (2021). How automated shuttles handle regular urban traffic? An article published at www.toi.no

6. Ryan, C., Murphy, F., \& Mullins, M. (2020). Spatial risk modelling of behavioural hotspots: Risk-aware path planning for autonomous vehicles. Transportation Research Part A: Policy and Practice, 134, 152-163. https:// doi.org/10.1016/j.tra.2020.01.024

7. Anderson, J. M., Kalra, N., Stanley, K. D., Sorensen, P., Samaras, C., \& Oluwatola, T. A. (2016). Autonomous vehicle technology — A guide for policymakers. Research report RR-443-2-RC, RAND Corporation. https://doi.org/10. 7249/RR443-2

8. Kalra, N., \& Paddock, S. M. (2016). Driving to safety—How many miles of driving would it take to demonstrate autonomous vehicle reliability? Research report RR-1478-RC, RAND Corporation. https://doi.org/10.7249/ RR1478 
9. Litman, T. (2020). Autonomous vehicle implementation predictions-implications for transport planning. Victoria Transport Policy Institute. https:// www.vtpi.org/avip.pdf

10. Thompson, J., Read, G. J. M., Wijnands, J. S., \& Salmon, P. M. (2020). The perils of perfect performance; considering the effects of introducing autonomous vehicles on rates of car vs cyclist conflict. Ergonomics, 63(8), 981-996. https://doi.org/10.1080/00140139.2020.173932

11. Ackermann, C., Beggiato, M., Bluhm, L.F., Löw, A., \& Krems, J. F. (2019). Deceleration parameters and their applicability as informal communication signal between pedestrians and automated vehicles. Transportation Research Part F: Traffic Psychology and Behaviour, 62, 757-768. https://doi. org/10.1016/j.trf.2019.03.006

12. Rasouli, A., \& Tsotsos, J. K. (2020). Autonomous vehicles that interact with pedestrians: A survey of theory and practice. IEEE Transactions on Intelligent Transportation Systems, 21(3), 900-918. https://doi.org/10.1109/TITS. 2019.2901817

13. Tarko, A. (2020). Measuring road safety with surrogate events. Elsevier.

14. Rovira, E., McLaughlin, A. C., Pak, R., \& High, L. (2019). Looking for age differences in self-driving vehicles: examining the effects of automation reliability, driving risk, and physical impairment on trust. Frontiers in Psychology. https://doi.org/10.3389/fpsyg.2019.00800

15. Vissers, L., van der Kint, S., van Schagen, I., \& Hagenzieker, M. (2016). Safe interaction between cyclists, pedestrians and automated vehicles - What do we know and what do we need to know? Research report R-2016-16, SWOV https://www.swov.nl/publicatie/safe-interaction-between-cyclists-pedes trians-and-automated-vehicles

16. Stanton, N. A., Eriksson, A., Banks, V. A., \& Hancock, P. A. (2020). Turing in the driver's seat: Can people distinguish between automated and manually driven vehicles? Human Factors and Ergonomics in Manufacturing \& Service Industries, 30, 418-425. https://doi.org/10.1002/hfm.20864

17. Bazilinsky, P., Sakuma, T., \& de Winter, J. (2021). What driving style makes pedestrians think a passing vehicle is driving automatically? Applied Ergonomics. https://doi.org/10.1016/j.apergo.2021.103428

18. Montoro, L., Useche, S. A., Alonso, F., Lijarcio, I., Bosó-Seguí, P., \& MartiBelda, A. (2019). Perceived safety and attributed value as predictors of the intention to use autonomous vehicles: A national study with Spanish drivers. Safety Science, 120, 865-876. https://doi.org/10.1016/j.ssci.2019.07. 041

19. Fraade-Blanar, L, Blumenthal, M.S., Anderson. J. M., \& Kalra, N. (2018). Measuring automated vehicle safety: Forging a framework. Research report RR-2662-RC, RAND Corporation. https://doi.org/10.7249/RR2662

20. Norden, J., O'Kelly, M., \& Sinha, A. (2020). Efficient black-box assessment of autonomous vehicle safety. Project report. Retrieved June 15, 2020, from https://arxiv.org/abs/1912.03618v2

21. Schoettle, B., \& Sivak, M. (2015). A preliminary analysis of real-world crashes involving self-driving vehicles. The University of Michigan Report No. UMTRI-2015-34. Retrieved July 20, 2020, from http://umich.edu/ umtri swt/PDF/UMTRI-2015-34.pdf

22. Morando, M. M., Tian, Q., Truong, L. T., \& Vu, H. L. (2018). Studying the safety impact of autonomous vehicles using simulation-based surrogate safety measures. Journal of Advanced Transportation. https://doi.org/10. 1155/2018/6135183

23. Pokorny, P. Skender, B., Biørnskau, T., \& Johnsson, E. (2021). Performance of automated shuttles at signalized intersections. TØI report 1822/2021. https://www.toi.no/publications/performance-of-automated-shuttles-atsignalised-intersections-article36784-29.html

24. Claburn, T. (2019). Literally braking news: Two people hurt as not one but two self-driving space-age buses go awry. On-line article. Retrieved July 20, 2020, from https://www.theregister.com/2019/07/19/selfdriving_bus_ injuries/

25. National Transportation Safety Board. (2019). Low-speed collision between truck-tractor and autonomous shuttle. Accident Report. Retrieved July 20, 2020, from https://www.ntsb.gov/investigations/AccidentReports/Repor ts/HAB1906.pdf
26. MacInnes, P. (2021). Toyota pauses Paralympics self-driving buses after one hits visually impaired athlete. The Guardian. https://www.theguardian. com/technology/2021/aug/28/toyota-pauses-paralympics-self-drivingbuses-after-one-hits-visually-impaired-athlete

27. Tom Bateman \& Reuters. (2021). Tokyo 2020 driverless buses lose self-driving functions after hitting Paralympic athlete. Euronews.com https://www. euronews.com/next/2021/08/30/toyota-halts-autonomous-e-palettebuses-after-one-hits-paralympic-athlete-in-tokyo-olympic

28. Jenssen, G. D., \& Moen, T. (2020). Trafikksikkerhet for automatiserte kjøretøy. https://www.sintef.no/en/publications/publication/1879225/

29. Azad, M., Hoseinzadeh, N., Brakewood, C., Cherry, Ch. R., \& Han, L. D. (2019). Fully autonomous buses: A literature review and future research directions. Journal of Advanced Transportation. https://doi.org/10.1155/ 2019/4603548

30. Salonen, A. O. (2018). Passenger's subjective traffic safety, in-vehicle security and emergency management in the driverless shuttle bus in Finland. Transport Policy, 61, 106-110. https://doi.org/10.1016/j.tranpol.2017.10. 011

31. Bjørnskau, T. (2021). Presentation of the Autobus project and field survey results. AUTOBUS final seminar, April 2021 (online). https://www.toi.no/ autobus/final-seminar/

32. Boersma, R., Van Arem, B., \& Rieck, F. (2018). Application of driverless electric automated shuttles for public transport in villages: The case of Appelscha. World Electric Vehicle Journal. https://doi.org/10.3390/wevj9 010015

33. Distler, V., Lallemand, C., \& Bellet, T. (2018). Acceptability and acceptance of autonomous mobility on demand: The impact of an immersive experience. In Proceedings of the $2018 \mathrm{CHI}$ conference on human factors in computing systems. Paper No.: 612. https://doi.org/10.1145/3173574. 3174186

34. Eden, G., Nanchen, B., Ramseyer, R., \& Evequoz, F. (2017). On the road with an autonomous passenger shuttle: Integration in public spaces. In Proceedings of the $2017 \mathrm{CHI}$ conference extended abstracts on human factors in computing systems. https://doi.org/10.1145/3027063.3053126

35. Straub, E. R., \& Schaefer, K. E. (2019). It takes two to Tango: Automated vehicles and human beings do the dance of driving - Four social considerations for policy. Transportation Research Part A: Policy and Practice, 122, 173-183. https://doi.org/10.1016/.t.tra.2018.03.005

36. Pokorny, P., \& Pitera, K. (2019). Truck-bicycle safety: An overview of methods of study, risk factors and research needs. European Transport Research Review. https://doi.org/10.1186/s12544-019-0371-7

37. Tønning, C., Madsen, T. K. O., Bahnsen, C. H., Moeslund, T. B., Agerholm, N., \& Lahrmann, H. S. (2017). Road user behaviour analyses based on video detections: Status and best practice examples from the RUBA software. In Proceedings of the 24th ITS World Congress. https://vbn.aau.dk/files/27356 9946/Road_user_behaviour_analyses_based_on_video_detections_ Status_and_best_practice_examples_from_the_RUBA_software.pdf

38. Laureshyn, A., Johnsson, C., Madsen, T., Varhelyi, A., de Goede, M., Svensson, Å., Saunier, N., \& van Haperen, W. (2017). Exploration of a method to validate surrogate safety measures with a focus on vulnerable road users. In Proceedings of the road safety \& simulation international conference, 17-19 October 2017. https://lup.lub.lu.se/record/04f53a04-0058-48109404-a2f294d3e 579

39 Biever, W., Angell, L., \& Seaman, S. (2019). Automated driving system collisions: Early lessons. Human Factors: The Journal of the Human Factors and Ergonomics Society. https://doi.org/10.1177/0018720819872034

\section{Publisher's Note}

Springer Nature remains neutral with regard to jurisdictional claims in published maps and institutional affiliations. 